\title{
Multi-Sensor Diversity in Decentralized Estimation
}

\author{
Alex S. Leong and Subhrakanti Dey
}

\begin{abstract}
This paper derives asymptotic expressions for the expected distortion in the decentralized estimation of a Gaussian source using multiple sensors, as the number of sensors becomes large. We consider a diversity scheme where only the sensor with the best channel sends their measurements to a fusion center, using the analog amplify and fowarding technique. For comparison, asymptotic expressions for a coherent multi-access scheme and an orthogonal access scheme are also derived.
\end{abstract}

\section{INTRODUCTION}

Wireless sensor networks have received much recent interest in the research community. Many different schemes for decentralized estimation of sources using multiple sensors have been proposed. One popular technique is analog amplify and forward [1], where sensors transmit a scaled version of their analog measurements to a fusion center, and has been shown to be optimal in some situations [2]. Analog forwarding under different multiple access schemes such as (coherent) multi-access [1], [3] and orthogonal access [4] have been studied.

In wireless communications theory the concept of multiuser diversity is well known [5], [6]. Multi-user diversity refers to different users experiencing good channel conditions at different times, and can be exploited in the following manner: For the problem of maximizing the sum rate subject to average power constraints, the optimal solution is to schedule the users such that at most only one user transmits, with this user being the one having the best channel conditions at that instance.

This paper will study the use of a similar diversity scheme in the estimation of a Gaussian source. In this scheme, which we will refer to as the multi-sensor diversity scheme, the sensor with the best channel will amplify and forward its measurement to the fusion center, while the other sensors do not transmit. In this paper we are interested in the asymptotic behaviour of such a scheme as the number of sensors $M$ goes to infinity. It is shown that in a symmetric sensor network the expected distortion decays to a non-zero limit at the rate $1 / \ln (M)$.

As a comparison we will also derive the expected distortion of the multi-access and orthognal access schemes, which decay at the rate $1 / M$ for large $M$. These results are similar to the existing asymptotic results for the distortion in the multi-access scheme [1], [7] and orthogonal access scheme [4], however the expected distortion is not considered explicitly in these works.

This work was supported by the Australian Research Council.

The authors are with the Department of Electrical and Electronic Engineering, University of Melbourne, Vic. 3010, Australia. (email: asleong@unimelb.edu.au; sdey@unimelb.edu.au)
The paper is organised as follows. Section II specifies our model. Section III derives the asymptotic behaviour for the multi-sensor diversity, multi-access, and orthogonal access schemes, followed by comparisons and discussions. Numerical studies are presented in Section IV.

\section{SYSTEM MODEL}

We wish to estimate a signal $\theta_{k}$ modelled as an i.i.d. Gaussian source with zero mean and variance $\sigma_{\theta}^{2}$, with $k$ representing the time index. The Gaussian source is measured by $M$ sensors with sensor $i$ having measurements

$$
y_{i, k}=\theta_{k}+v_{i, k}, i=1, \ldots, M
$$

with $v_{i, k}$ being i.i.d. Gaussian with zero mean and noise variance $\sigma_{i}^{2}$, with $v_{i, k}$ independent of $v_{j, k}$ for $i \neq j$. Let $g_{i, k}$ be the randomly time-varying channel gains from sensor $i$ to the fusion center, and $\alpha_{i, k}$ the amplification factors in the amplify and forward scheme. We assume that $g_{i, k}$ and $g_{j, k}$ are independent for $i \neq j$. The transmit power of sensor $i$ at time $k$ is defined as

$$
\gamma_{i, k}=\alpha_{i, k}^{2} \mathbb{E}\left[y_{i, k}^{2}\right]=\alpha_{i, k}^{2}\left(\sigma_{\theta}^{2}+\sigma_{i}^{2}\right)
$$

Different multiple access schemes for transmitting the sensor measurements to a fusion center will now be presented.

\section{A. Multi-sensor diversity scheme}

Let $g_{\max , k}=\max \left(g_{1, k}, \ldots, g_{M, k}\right)$, and $i^{*}$ the index of the corresponding sensor. Consider a scheme where only the sensor with the best channel transmits its measurement to the fusion center. The fusion center then receives

$$
z_{k}=\sqrt{g_{\max , k}} \alpha_{i^{*}, k}\left(\theta_{k}+v_{i^{*}, k}\right)+n_{k}
$$

where $n_{k}$ is i.i.d. Gaussian with zero mean and variance $\sigma_{n}^{2}$. Using the linear MMSE estimator, the mean squared error or distortion at time $k$ can be easily shown to be

$$
D_{k}=\left(\frac{1}{\sigma_{\theta}^{2}}+\frac{g_{\max , k} \alpha_{i^{*}, k}^{2}}{g_{\max , k} \alpha_{i^{*}, k}^{2} \sigma_{i^{*}}^{2}+\sigma_{n}^{2}}\right)^{-1}
$$

\section{B. Multi-access scheme}

In the multi-access scheme the sensors transmit their measurements to the fusion center using the amplify and forward technique over a multi-access channel, so the fusion center receives the sum

$$
z_{k}=\sum_{i=1}^{M} \sqrt{g_{i, k}} \alpha_{i, k}\left(\theta_{k}+v_{i, k}\right)+n_{k}
$$


The distortion at time $k$ is given by

$$
\begin{aligned}
D_{k} & =\left(\frac{1}{\sigma_{\theta}^{2}}+\frac{\left(\sum_{i=1}^{M} \sqrt{g_{i, k}} \alpha_{i, k}\right)^{2}}{\sum_{i=1}^{M} g_{i, k} \alpha_{i, k}^{2} \sigma_{i}^{2}+\sigma_{n}^{2}}\right)^{-1} \\
& =\frac{\sigma_{\theta}^{2}\left(\sum_{i=1}^{M} g_{i, k} \alpha_{i, k}^{2} \sigma_{i}^{2}+\sigma_{n}^{2}\right)}{\sum_{i=1}^{M} g_{i, k} \alpha_{i, k}^{2} \sigma_{i}^{2}+\sigma_{n}^{2}+\sigma_{\theta}^{2}\left(\sum_{i=1}^{M} \sqrt{g_{i, k}} \alpha_{i, k}\right)^{2}}
\end{aligned}
$$

\section{Orthogonal access scheme}

In the orthogonal access scheme the sensors transmit their measurements to the fusion center via orthogonal channels, so that the fusion center receives

$$
z_{i, k}=\sqrt{g_{i, k}} \alpha_{i, k}\left(\theta_{k}+v_{i, k}\right)+n_{i, k}, i=1, \ldots, M
$$

where $n_{i, k}$ is i.i.d. Gaussian with zero mean and variance $\sigma_{n}^{2}, \forall i$. The distortion at time $k$ is given by

$$
D_{k}=\left(\frac{1}{\sigma_{\theta}^{2}}+\sum_{i=1}^{M} \frac{g_{i, k} \alpha_{i, k}^{2}}{g_{i, k} \alpha_{i, k}^{2} \sigma_{i}^{2}+\sigma_{n}^{2}}\right)^{-1}
$$

\section{ASYMPTOTIC ANALYSIS}

We are interested in deriving asymptotic expressions for $\mathbb{E}\left[D_{k}\right]$ as $M \rightarrow \infty$, where the expectation is over the random channel gains $g_{i, k}$, for the three different schemes in Section II. By the i.i.d. (in time) nature of the models we will drop the subscript $k$. For analytical tractibility we will first analyze "symmetric" sensor networks with $\sigma_{i}^{2}=\sigma_{v}^{2}, \forall i$, and with the $g_{i}$ 's being identically distributed. See Section III-E for remarks on more general asymmetric situations. Apart from the multi-access scheme, for the other schemes we will need to assume a specific distribution in order to obtain precise asymptotic results. In these cases we will assume Rayleigh fading, though most of our analytical methods should be able to be adapted to other fading distributions.

Notation: For two functions $f(t)$ and $g(t)$, we will use the standard asymptotic notation (see e.g. [8]) and say that $f \sim g$ as $t \rightarrow t_{0}$, if $\frac{f(t)}{g(t)} \rightarrow 1$ as $t \rightarrow t_{0}$. It is well known that the asymptotic relation $\sim$ is retained under addition, multiplication and division.

Notation: Extending the use of the symbol $\sim$ to functions of random variables, for functions $f(t, \omega)$ and $g(t, \omega)$, we will also say that $f \sim g$ w.p.1 as $t \rightarrow t_{0}$, if $\frac{f(t, \bullet)}{g(t, \bullet)} \rightarrow$ 1 w.p. 1 as $t \rightarrow t_{0}$. For instance, if $X_{i}$ are i.i.d., then $\sum_{i=1}^{M} X_{i} \sim M \mathbb{E}\left[X_{1}\right]$ w.p. 1 as $M \rightarrow \infty$, which follows from the definition and the strong law of large numbers.

\section{A. Multi-sensor diversity scheme}

Let us use $\alpha_{i^{*}}=1$, and $\alpha_{j}=0, \forall j \neq i^{*}$. Then

$$
\begin{aligned}
D & =\left(\frac{1}{\sigma_{\theta}^{2}}+\frac{g_{\max }}{g_{\max } \sigma_{v}^{2}+\sigma_{n}^{2}}\right)^{-1} \\
& =\frac{\sigma_{\theta}^{2}\left(g_{\max } \sigma_{v}^{2}+\sigma_{n}^{2}\right)}{g_{\max }\left(\sigma_{v}^{2}+\sigma_{\theta}^{2}\right)+\sigma_{n}^{2}} \\
& =\frac{\sigma_{\theta}^{2} \sigma_{v}^{2}}{\sigma_{\theta}^{2}+\sigma_{v}^{2}}\left(1+\frac{\frac{\sigma_{n}^{2} \sigma_{\theta}^{2}}{\sigma_{v}^{2}\left(\sigma_{\theta}^{2}+\sigma_{v}^{2}\right)}}{g_{\max }+\frac{\sigma_{n}^{2}}{\sigma_{\theta}^{2}+\sigma_{v}^{2}}}\right)
\end{aligned}
$$

Therefore

$$
\mathbb{E}[D]=\frac{\sigma_{\theta}^{2} \sigma_{v}^{2}}{\sigma_{\theta}^{2}+\sigma_{v}^{2}} \mathbb{E}\left[1+\frac{\frac{\sigma_{n}^{2} \sigma_{\theta}^{2}}{\sigma_{v}^{2}\left(\sigma_{\theta}^{2}+\sigma_{v}^{2}\right)}}{g_{\max }+\frac{\sigma_{n}^{2}}{\sigma_{\theta}^{2}+\sigma_{v}^{2}}}\right]
$$

Considering Rayleigh fading, we have the following result.

Lemma 1: Suppose the $g_{i}$ 's are exponentially distributed with mean $1 / \lambda$, and let $b>0$ be a constant. Then

$$
\mathbb{E}\left[\frac{1}{g_{\max }+b}\right] \sim \frac{\lambda}{\lambda b+\ln M} \sim \frac{\lambda}{\ln (M)} \text { as } M \rightarrow \infty
$$

See Appendix for the proof.

Using Lemma 1, we then have

$$
\begin{aligned}
\mathbb{E}[D] & \sim \frac{\sigma_{\theta}^{2} \sigma_{v}^{2}}{\sigma_{\theta}^{2}+\sigma_{v}^{2}}\left[1+\frac{\sigma_{n}^{2} \sigma_{\theta}^{2}}{\sigma_{v}^{2}\left(\sigma_{\theta}^{2}+\sigma_{v}^{2}\right)} \frac{\lambda}{\ln (M)+\frac{\lambda \sigma_{n}^{2}}{\sigma_{\theta}^{2}+\sigma_{v}^{2}}}\right] \\
& \sim \frac{\sigma_{\theta}^{2} \sigma_{v}^{2}}{\sigma_{\theta}^{2}+\sigma_{v}^{2}}\left[1+\frac{\sigma_{n}^{2} \sigma_{\theta}^{2}}{\sigma_{v}^{2}\left(\sigma_{\theta}^{2}+\sigma_{v}^{2}\right)} \frac{\lambda}{\ln (M)}\right]
\end{aligned}
$$

Hence as $M \rightarrow \infty$, the expected distortion goes to $\frac{\sigma_{\theta}^{2} \sigma_{v}^{2}}{\sigma_{\theta}^{2}+\sigma_{v}^{2}}$ at the rate $1 / \ln (M)$.

\section{B. Multi-access scheme}

For purposes of comparison with the diversity scheme, let us use here the scaling $\alpha_{i}=1 / \sqrt{M}, \forall i$, which will give the same long term average transmit power usage. Then

$$
\begin{aligned}
D & =\frac{\sigma_{\theta}^{2}\left(\sigma_{v}^{2} \frac{1}{M} \sum_{i=1}^{M} g_{i}+\sigma_{n}^{2}\right)}{\sigma_{v}^{2} \frac{1}{M} \sum_{i=1}^{M} g_{i}+\sigma_{n}^{2}+M \sigma_{\theta}^{2}\left(\frac{1}{M} \sum_{i=1}^{M} \sqrt{g_{i}}\right)^{2}} \\
& \sim \frac{\sigma_{\theta}^{2}\left(\sigma_{v}^{2} \mathbb{E}\left[g_{1}\right]+\sigma_{n}^{2}\right)}{\sigma_{v}^{2} \mathbb{E}\left[g_{1}\right]+\sigma_{n}^{2}+M \sigma_{\theta}^{2}\left(\mathbb{E}\left[\sqrt{g_{1}}\right]\right)^{2}} \text { a.s. }
\end{aligned}
$$

provided the expectations $\mathbb{E}\left[g_{1}\right]$ and $\mathbb{E}\left[\sqrt{g_{1}}\right]$ exist, where the last line comes from applying the strong law of large numbers and the definition and properties of $\sim$. Since $D$ is always bounded, we can then use results on uniform integrability, e.g. [9], to conclude that

$$
\begin{aligned}
\mathbb{E}[D] & \sim \frac{\sigma_{\theta}^{2}\left(\sigma_{v}^{2} \mathbb{E}\left[g_{1}\right]+\sigma_{n}^{2}\right)}{\sigma_{v}^{2} \mathbb{E}\left[g_{1}\right]+\sigma_{n}^{2}+M \sigma_{\theta}^{2}\left(\mathbb{E}\left[\sqrt{g_{1}}\right]\right)^{2}} \\
& \sim \frac{\sigma_{v}^{2} \mathbb{E}\left[g_{1}\right]+\sigma_{n}^{2}}{M\left(\mathbb{E}\left[\sqrt{g_{1}}\right]\right)^{2}}
\end{aligned}
$$

Thus the expected distortion decays to zero at the rate $1 / M$ as $M \rightarrow \infty$, similar to the scaling behaviour for the distortion derived in [7].

\section{Orthogonal access scheme}

Let us again use here the scaling $\alpha_{i}=1 / \sqrt{M}, \forall i$. Then

$$
\begin{aligned}
D & =\frac{1}{\frac{1}{\sigma_{\theta}^{2}}+\sum_{i=1}^{M} \frac{g_{i} / M}{g_{i} \sigma_{v}^{2} / M+\sigma_{n}^{2}}} \\
& =\frac{1}{\frac{1}{\sigma_{\theta}^{2}}+\sum_{i=1}^{M} \frac{g_{i}}{g_{i} \sigma_{v}^{2}+M \sigma_{n}^{2}}} \\
& \sim \frac{1}{\frac{1}{\sigma_{\theta}^{2}}+M \mathbb{E}\left[\frac{g_{1}}{g_{1} \sigma_{v}^{2}+M \sigma_{n}^{2}}\right]} \text { a.s. }
\end{aligned}
$$


provided the expectation $\mathbb{E}\left[g_{1} /\left(g_{1} \sigma_{v}^{2}+\sigma_{n}^{2}\right)\right]$ (and hence $\left.\mathbb{E}\left[g_{1} /\left(g_{1} \sigma_{v}^{2}+M \sigma_{n}^{2}\right)\right]\right)$ exists, where the last line now comes from using a strong law of large numbers for triangular arrays [10], and the definition and properties of $\sim$. Hence by uniform integrability

$$
\mathbb{E}[D] \sim \frac{1}{\frac{1}{\sigma_{\theta}^{2}}+M \mathbb{E}\left[\frac{g_{1}}{g_{1} \sigma_{v}^{2}+M \sigma_{n}^{2}}\right]}
$$

If we now assume Rayleigh fading, with $g_{i}$ being exponentially distributed with mean $1 / \lambda$, then

$$
\begin{aligned}
\mathbb{E} & {\left[\frac{g_{1}}{g_{1} \sigma_{v}^{2}+M \sigma_{n}^{2}}\right]=\mathbb{E}\left[\frac{1}{\sigma_{v}^{2}}\left(1-\frac{M \sigma_{n}^{2} / \sigma_{v}^{2}}{g_{1}+M \sigma_{n}^{2} / \sigma_{v}^{2}}\right)\right] } \\
& =\frac{1}{\sigma_{v}^{2}}\left[1-\frac{\lambda M \sigma_{n}^{2}}{\sigma_{v}^{2}} \exp \left(\frac{\lambda M \sigma_{n}^{2}}{\sigma_{v}^{2}}\right) E_{1}\left(\frac{\lambda M \sigma_{n}^{2}}{\sigma_{v}^{2}}\right)\right] \\
& \sim \frac{1}{\sigma_{v}^{2}}\left(\frac{\sigma_{v}^{2}}{\lambda M \sigma_{n}^{2}}-\frac{2 \sigma_{v}^{4}}{\lambda^{2} M^{2} \sigma_{n}^{4}}\right)
\end{aligned}
$$

where $E_{1}($.$) is the exponential integral, and we have used$ the asymptotic expansion [11]

$$
E_{1}(z) \sim \frac{e^{-z}}{z}\left(1-\frac{1}{z}+\frac{2}{z^{2}}-+\ldots\right)
$$

Hence for Rayleigh fading

$$
\begin{aligned}
\mathbb{E}[D] & \sim \frac{1}{\frac{1}{\sigma_{\theta}^{2}}+\frac{1}{\lambda \sigma_{n}^{2}}-\frac{2 \sigma_{v}^{2}}{\lambda^{2} M \sigma_{n}^{4}}} \\
& \sim \frac{1}{\frac{1}{\sigma_{\theta}^{2}}+\frac{1}{\lambda \sigma_{n}^{2}}}+\frac{2 \sigma_{v}^{2}}{M \lambda^{2} \sigma_{n}^{4}} \frac{1}{\left(\frac{1}{\sigma_{\theta}^{2}}+\frac{1}{\lambda \sigma_{n}^{2}}\right)^{2}}
\end{aligned}
$$

which converges to $\left(\frac{1}{\sigma_{\theta}^{2}}+\frac{1}{\lambda \sigma_{n}^{2}}\right)^{-1}$ at the rate $1 / M$. The limit $\left(\frac{1}{\sigma_{\theta}^{2}}+\frac{1}{\lambda \sigma_{n}^{2}}\right)^{-1}$ for $D$ as $M \rightarrow \infty$ was also previously shown in [4], though the rate of convergence was not derived.

\section{Comparisons and discussions}

The limit $\frac{\sigma_{\theta}^{2} \sigma_{v}^{2}}{\sigma_{\theta}^{2}+\sigma_{v}^{2}}=\left(\frac{1}{\sigma_{\theta}^{2}}+\frac{1}{\sigma_{v}^{2}}\right)^{-1}$ in the multi-sensor diversity scheme corresponds to the distortion that can be achieved with a single sensor with estimation performed at that sensor, i.e. no further analog forwarding to a fusion center. We note also that the limit $\left(\frac{1}{\sigma_{\theta}^{2}}+\frac{1}{\lambda \sigma_{n}^{2}}\right)^{-1}$ in the orthogonal scheme using $\alpha_{i}=1 / \sqrt{M}, \forall i$ is different from the limit in the diversity scheme. Under the choices of $\alpha_{i}$ in this paper, the expected distortion goes to zero only in the multi-access scheme. ${ }^{1}$

In terms of speed of convergence, the rate $1 / M$ is achieved in the multi-access and orthogonal schemes. On the other hand, we get a slower convergence rate of $1 / \ln (M)$ in the diversity scheme. A similar $1 / \ln (M)$ rate is achieved when sensor measurements are transmitted to a fusion center digitally using separate source/channel coding, e.g. as in the CEO problem [1], [12].

Finally, in regards to implementation, the multi-access scheme requires that we add the measurements coherently as in (1), which may be difficult to achieve for large sensor

\footnotetext{
${ }^{1}$ However if e.g. we use $\alpha_{i}=1, \forall i$, then the expected distortion will also go to zero in the orthogonal scheme.
}

networks. The orthogonal access scheme does not require as much synchronization between sensors [4], but each sensor will require its own orthogonal channel. The multi-sensor diversity scheme doesn't have these issues, though it will still require the fusion center to determine which sensor has the best channel, with this information then fed back to the sensors.

\section{E. General sensor noise variances}

We will now consider the case where the sensor noise variances $\sigma_{i}^{2}, i=1, \ldots, M$ are not necessarily identical, though for analytical tractibility the fading channels are still assumed to be i.i.d. across sensors. The idea is to obtain upper and lower bounds on the expected distortion which asymptotically will have the same scaling behaviour, a similar method was used in [13] in the context of linear state estimation. We suppose that the sensor noise variances can be bounded from both above and below, i.e.

$$
0<\sigma_{\min }^{2} \leq \sigma_{i}^{2} \leq \sigma_{\max }^{2}<\infty, \forall i
$$

Then we note in all the different schemes considered here (multi-sensor diversity, multi-access, orthogonal accesss), $D$ is an increasing function of $\sigma_{i}^{2}$ for all $i$. Hence we can upper and lower bound $D$ with the symmetric results using $\sigma_{i}^{2}=$ $\sigma_{\text {max }}^{2}, \forall i$ and $\sigma_{i}^{2}=\sigma_{\text {min }}^{2}, \forall i$ respectively.

Then in the multi-access scheme, since the upper and lower bounds both converge to zero at the rate $1 / M$, the general situation will also have the same scaling behaviour as the bounds. Simlarly, for the orthogonal access scheme we have upper and lower bounds both converging to $\left(\frac{1}{\sigma_{\theta}^{2}}+\right.$ $\left.\frac{1}{\lambda \sigma_{n}^{2}}\right)^{-1}$ at the rate $1 / M$, and so the general situation will also do so. On the other hand, it can be shown that in general the multi-sensor diversity scheme will not converge to any limit as $M \rightarrow \infty$, by using an argument similar to one in [13].

\section{NUMERICAL STUDIES}

Consider a situation with $\sigma_{\theta}^{2}=1, \sigma_{v}^{2}=0.2, \sigma_{n}^{2}=0.1$, and let $g_{i}, \forall i$ be exponentially distributed with mean $1 / 2$. Note that then $\mathbb{E}\left[\sqrt{g_{i}}\right]=\sqrt{\pi / 8},\left(\frac{1}{\sigma_{\theta}^{2}}+\frac{1}{\sigma_{v}^{2}}\right)^{-1}=0.1667$, and $\left(\frac{1}{\sigma_{\theta}^{2}}+\frac{1}{\lambda \sigma_{n}^{2}}\right)^{-1}=0.1667$.

In Fig. 1 we compare between the simulated expected distortion (averaging over 100000 iterations) and the asymptotic expression (2) for the multi-sensor diversity scheme, for different numbers of sensors $M$. In Fig. 2 we compare between the simulated expected distortion and the asymptotic expression (3) for the multi-access scheme. In Fig. 3 we compare between the simulated expected distortion and the asymptotic expression (4) for the orthogonal access scheme. In each case, the validity of the respective asymptotic expressions for large $M$ is confirmed.

\section{CONCLUSION}

This paper has studied the asymptotic behaviour of a multi-sensor diversity scheme for the decentralized estimation of an i.i.d. Gaussian source. We have shown that the rate of decay is $1 / \ln (M)$ as the number of sensors $M$ becomes 


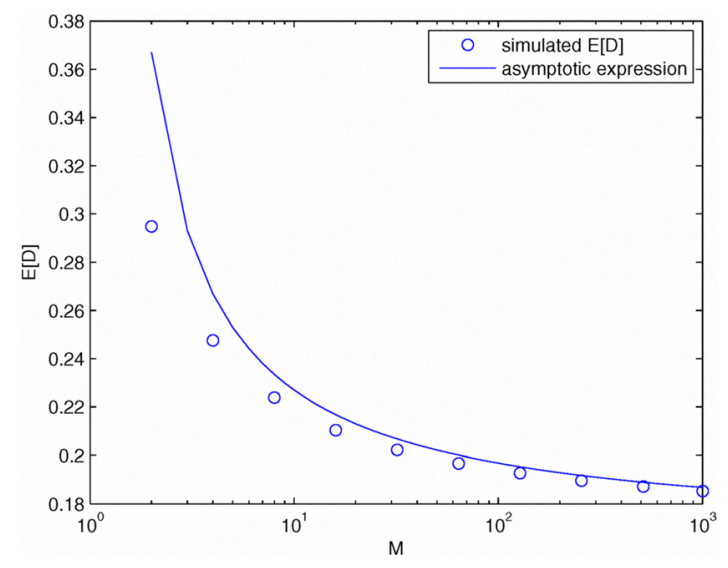

Fig. 1. Multi-sensor diversity scheme. Comparison between simulated expected distortion and asymptotic expression.

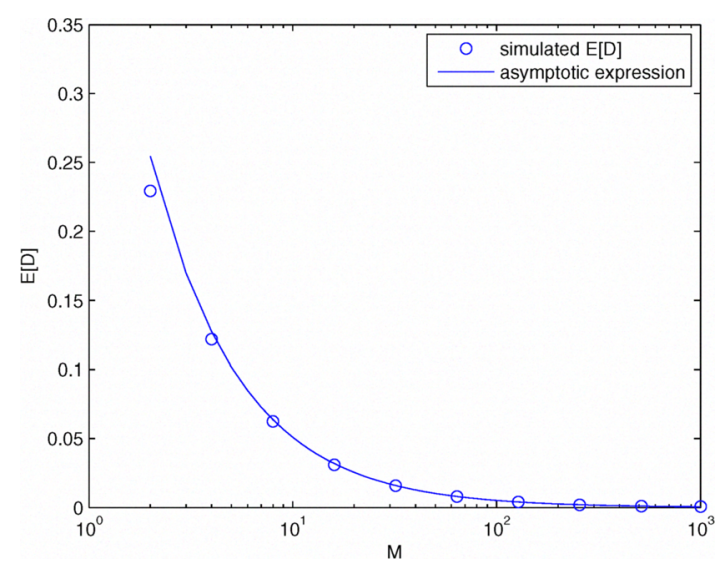

Fig. 2. Multi-access scheme. Comparison between simulated expected distortion and asymptotic expression.

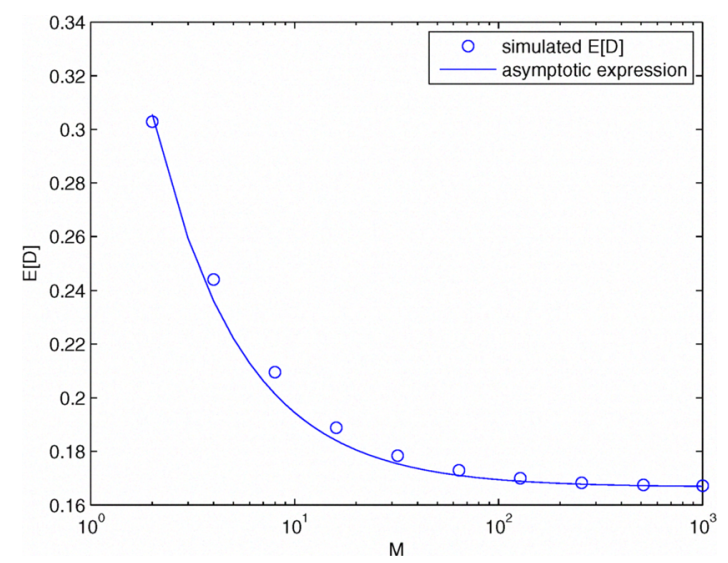

Fig. 3. Orthogonal access scheme. Comparison between simulated expected distortion and asymptotic expression. large. In comparison, the multi-access and orthogonal access schemes have been shown to have a rate of decay of $1 / M$. As an extension of this work, a distributed version of the multisensor diversity scheme called the channel-aware ALOHA scheme, based on ideas of [14] can be analyzed. Furthermore, optimal power allocation in the multi-sensor diversity and channel-aware ALOHA scheme, and its performance in the case of large numbers of sensors, has also be studied. These results will be presented in future work.

\section{APPENDIX}

\section{A. Proof of Lemma 1}

Proof: The maximum of $M$ i.i.d. exponential random variables with mean $1 / \lambda$, has cumulative distribution function

$$
F(x)=\left(1-e^{-\lambda x}\right)^{M}
$$

and hence the probability density function

$$
p(x)=M\left(1-e^{-\lambda x}\right)^{M-1} \lambda e^{-\lambda x} .
$$

We wish to find the large $M$ behaviour of

$$
\begin{aligned}
\mathbb{E}\left[\frac{1}{X+b}\right] & =M \int_{0}^{\infty} \frac{\left(1-e^{-\lambda x}\right)^{M-1} \lambda e^{-\lambda x}}{x+b} d x \\
& =M \int_{0}^{\infty} \frac{e^{-M t}}{b-\frac{1}{\lambda} \ln \left(1-e^{-t}\right)} d t
\end{aligned}
$$

where in the second line we used the substitution $e^{-t}=1-$ $e^{-\lambda x}$. To determine the asymptotic behaviour of the integral

$$
\int_{0}^{\infty} \frac{e^{-M t}}{b-\frac{1}{\lambda} \ln \left(1-e^{-t}\right)} d t
$$

we will use a Tauberian theorem for the Laplace transform, see p.445 of [15] or p.248 of [16], which in our notation says that if $f(t) \geq 0,0 \leq \rho<\infty$, and $L(t)$ is a slowly varying function at infinity, then each of the relations

$$
\int_{0}^{\infty} e^{-M t} f(t) d t \sim M^{-\rho} L\left(\frac{1}{M}\right) \text { as } M \rightarrow \infty
$$

and

$$
\int_{0}^{t} f(\tau) d \tau \sim \frac{t^{\rho} L(t)}{\Gamma(\rho+1)} \text { as } t \rightarrow 0
$$

implies the other.

Thus we can study first the asymptotic behaviour of

$$
\int_{0}^{t} \frac{1}{b-\frac{1}{\lambda} \ln \left(1-e^{-\tau}\right)} d \tau=\lambda \int_{0}^{t} \frac{1}{\lambda b-\ln \left(1-e^{-\tau}\right)} d \tau
$$

as $t \rightarrow 0$. Using an integration by parts, we obtain

$$
\begin{aligned}
& \int_{0}^{t} \frac{1}{\lambda b-\ln \left(1-e^{-\tau}\right)} d \tau= \\
& \frac{t}{\lambda b-\ln \left(1-e^{-t}\right)}-\int_{0}^{t} \frac{\tau e^{-\tau}}{\left(1-e^{-\tau}\right)\left(\lambda b-\ln \left(1-e^{-\tau}\right)\right)^{2}} d \tau
\end{aligned}
$$


Next, it may be verified that $\tau e^{-\tau} /\left(1-e^{-\tau}\right) \leq 1$, and that $1 /\left(\lambda b-\ln \left(1-e^{-\tau}\right)\right)^{2}$ is an increasing function of $\tau$. Then

$$
\begin{aligned}
\mid \int_{0}^{t} & \frac{\tau e^{-\tau}}{\left(1-e^{-\tau}\right)\left(\lambda b-\ln \left(1-e^{-\tau}\right)\right)^{2}} d \tau \mid \\
\quad & \int_{0}^{t} \frac{\tau e^{-\tau}}{\left(1-e^{-\tau}\right)\left(\lambda b-\ln \left(1-e^{-\tau}\right)\right)^{2}} d \tau \\
& \leq \int_{0}^{t} \frac{1}{\left(\lambda b-\ln \left(1-e^{-\tau}\right)\right)^{2}} d \tau \\
& \leq \frac{t}{\left(\lambda b-\ln \left(1-e^{-t}\right)\right)^{2}} \\
& =o\left(\frac{t}{\lambda b-\ln \left(1-e^{-t}\right)}\right) \text { as } t \rightarrow 0
\end{aligned}
$$

and so

$$
\begin{aligned}
\lambda \int_{0}^{t} \frac{1}{\lambda b-\ln \left(1-e^{-\tau}\right)} d \tau & \sim \frac{\lambda t}{\lambda b-\ln \left(1-e^{-t}\right)} \\
& \sim \frac{\lambda t}{\lambda b-\ln (t)}=\frac{\lambda t}{\lambda b+\ln (1 / t)}
\end{aligned}
$$

as $t \rightarrow 0$. With $L(t)=\frac{\lambda}{\lambda b+\ln (1 / t)}$ (which can be easily shown to be slowly varying) and $\rho=1$, we thus have by the Tauberian theorem that

$$
\begin{aligned}
M \int_{0}^{\infty} \frac{e^{-M t}}{b-\frac{1}{\lambda} \ln \left(1-e^{-t}\right)} d t & \sim M \times M^{-1} \times \frac{\lambda}{\lambda b+\ln (M)} \\
& \sim \frac{\lambda}{\ln (M)} \text { as } M \rightarrow \infty
\end{aligned}
$$

\section{ACKNOWLEDGMENT}

The authors thank Prof. Barry Hughes of the Department of Mathematics and Statistics, University of Melbourne, for his help on the proof of Lemma 1.

\section{REFERENCES}

[1] M. Gastpar and M. Vetterli, "Source-channel communication in sensor networks," Springer Lecture Notes in Computer Science, vol. 2634, pp. 162-177, Apr. 2003

[2] M. Gastpar, "Uncoded transmission is exactly optimal for a simple Gaussian sensor network," IEEE Trans. Inform. Theory, vol. 54, no. 11, pp. 5247-5251, Nov. 2008.

[3] J.-J. Xiao, S. Cui, Z.-Q. Luo, and A. J. Goldsmith, "Linear coherent decentralized estimation," IEEE Trans. Signal Processing, vol. 56, no. 2, pp. 757-770, Feb. 2008.

[4] S. Cui, J.-J. Xiao, A. Goldsmith, Z.-Q. Luo, and H. V. Poor, "Estimation diversity and energy efficiency in distributed sensing," IEEE Trans. Signal Processing, vol. 55, no. 9, pp. 4683-4695, Sept. 2007.

[5] R. Knopp and P. A. Humblet, "Information capacity and power control in single-cell multiuser communications," in Proc. IEEE. Int. Conf. Commun., Seattle, WA, June 1995, pp. 331-335.

[6] P. Viswanath, D. N. C. Tse, and R. Laroia, "Opportunistic beamforming using dumb antennas," IEEE Trans. Inform. Theory, vol. 48, no. 6, pp. 1277-1294, June 2002.

[7] M. Gastpar and M. Vetterli, "Power, spatio-temporal bandwidth, and distortion in large sensor networks," IEEE J. Select. Areas Commun., vol. 23, no. 4, pp. 745-754, Apr. 2005.

[8] F. W. J. Olver, Asymptotics and Special Functions. New York: Academic Press, 1974.

[9] G. R. Grimmett and D. R. Stirzaker, Probability and Random Processes, 3rd ed. Oxford, Great Britain: Oxford University Press, 2001.

[10] T.-C. Hu, F. Móricz, and R. L. Taylor, "Strong laws of large numbers for arrays of rowwise independent random variables," Acta Math. Hung., vol. 54, no. 1-2, pp. 153-162, 1989.
[11] M. Abramowitz and I. A. Stegun, Handbook of Mathematical Functions. New York: Dover Publications, 1965.

[12] T. Berger, Z. Zhang, and H. Viswanathan, "The CEO problem," IEEE Trans. Inform. Theory, vol. 42, no. 3, pp. 887-902, May 1996.

[13] A. S. Leong, S. Dey, and J. S. Evans, "Asymptotics and power allocation for state estimation over fading channels," IEEE Trans. Aerosp. Electron. Syst., to appear. Available at http://arxiv.org/abs/0803.3850.

[14] X. Qin and R. A. Berry, "Distributed approaches for exploiting multiuser diversity in wireless networks," IEEE Trans. Inform. Theory, vol. 52, no. 2, pp. 392-413, Feb. 2006.

[15] W. Feller, An Introduction to Probability Theory and Its Applications: Volume II, 2nd ed. New York: John Wiley \& Sons, 1971.

[16] B. D. Hughes, Random Walks and Random Environments: Volume 1. New York: Oxford University Press, 1995. 